\title{
Parkinson's disease and depression: psychometric properties of the Beck Depression Inventory
}

\author{
BONNIE E LEVIN,* MARIA M LLABRE, $\dagger$ WILLIAM J WEINER* \\ From the Departments of Neurology* and Psychology, $†$ University of Miami, Miami, USA
}

SUMmaRY Although the Beck Depression Inventory (BDI) is one of the most frequently employed measures of depression in Parkinson's disease, the somatic items included in the scale raise questions about its ability to differentiate depression from manifestations of the disease. The internal consistency and validity of the BDI as a measure of depression in a sample of 119 Parkinson's disease patients and 76 controls were studied. Results from an item analysis suggested that depression in Parkinson's disease patients is not a somatic artifact. Internal consistency reliability within the Parkinson's disease group was high. The factorial validity of the BDI was confirmed in both Parkinson's disease and control subjects. The results also indicated that Parkinsonian symptoms of depression can be measured separately from symptoms of the disease. These data suggest that the BDI including the somatic items is a reliable and valid measure of depression in Parkinson's disease and control subjects.

Estimates of the incidence of depression in Parkinson's disease range from $37 \%$ to $90 \% .^{12}$ The variability in these estimates may be due in part to differences in the measures used to assess depression. The Beck Depression Inventory (BDI) has been used in over 500 reported studies ${ }^{3}$ and is currently gaining increasing recognition as a reliable measure of depression symptomatology in Parkinson's disease patients. $^{4-8}$

However, one major criticism of this scale is that it is heavily weighted toward somatic complaints, ${ }^{9}$ making it difficult to distinguish between symptoms of depression and symptoms of the disease. ${ }^{10}$ It can be argued that Parkinsonian patients are more likely to endorse somatic items on depression scales because of the characteristics of the disease and not necessarily because they are depressed. Patients with Parkinson's disease often complain of lack of energy, difficulty in performing routine tasks, fatigue and sleep disruption.

The assertion that the somatic content of the BDI makes it difficult to separate symptoms of depression from manifestations of Parkinson's disease is logical. However, there are no empirical studies which have

Address for reprint requests: Bonnie E Levin, PhD, University of Miami, School of Medicine, Department of Neurology (D4-5), PO Box 016960, Miami, Florida 33101, USA.

Received 11 December 1987 and in revised form 10 June 1988. Accepted 20 June 1988. demonstrated that the somatic items detract from the validity and reliability of the BDI as a measure of depression in Parkinson's disease patients. The purpose of this study was to determine whether the somatic items on the BDI detracted from its internal consistency and validity as a measure of depression with Parkinson's disease patients.

\section{Methods}

\section{Subjects}

One hundred and nineteen patients with idiopathic Parkinson's disease served as the experimental group. The Parkinson's disease group consisted of 81 male and 38 female volunteers, from the Movement Disorders Clinic at the University of Miami. Nine of the subjects recruited for the study refused to participate. Those with a history of cardiovascular disease, CVA, encephalitis, head trauma or other neurological problems were excluded. All patients were receiving antiparkinsonian medication. Mean age was 65 years and mean level of education was 14 years of schooling. Mean stage (Hoehn and Yahr) and duration of illness were 2.2 and 6.6 years, respectively. Mean age of onset was 59 years. At the time of testing $12(11 \%)$ of the patients were taking antidepressant medication.

The control group consisted of 76 subjects, 29 males and 47 females, who were spouses and volunteers from a retirement community. Control subjects had a mean age of 68 years and 13 years of schooling. All of the control subjects recruited for the study agreed to participate. None of the control subjects had a history of either neurological or psychiatric disease. All subjects gave informed consent. 


\section{Procedures}

Subjects in the Parkinson's disease group were first interviewed and examined by a neurologist. Degree of disability was assessed using a modified Columbia University motor disability scale. As part of this scale, subjects were assessed for rigidity (R), grades $0-4$; tremor $(\mathrm{T}), 0-4$; and bradykinesia (B), 0-4.

All subjects were administered two indices of depression: The $\mathrm{BDI}^{11}$ and the depression subscales of the Millon Behavioral Health Inventory (MBHI). ${ }^{12}$ The BDI is a 21 item scale originally derived from clinical observations of depressed psychiatric patients. ${ }^{3}$ Items are rated from 0 to 3 and the resulting scores are summed across items to yield an overall measure of the intensity of depressive symptomatology. For purposes of this study, items were classified as somatic or nonsomatic. This classification was ad hoc and based on a logical analysis of the items. Two subscales were constructed: one based on the seven somatic items (S) and the other based on the 14 nonsomatic items (NS).

The MBHI subscales were used as criteria of depression. The MBHI is a 150 item, true/false questionnaire with two subscales directed towards measuring depression in a medical population. The first subscale, premorbid pessimism (PP), was designed to assess an individual's premorbid attitude toward the way life has treated him/her in the past and is assumed to measure stable dispositional characteristics. The second subscale, future despair (FD), was designed to assess a patient's current feelings regarding future plans.

The scales on the MBHI were chosen as criterion measures of depression for several reasons. First, the scales were designed to assess two different aspects of depression: longstanding depressive personality traits and recent situational reaction. Second, the items were not designed to be symptom oriented, eliminating the overlap of items containing somatic as well as depressive traits. Third, as previously stated, the scales were normed on medical samples rather than psychiatrically impaired individuals. Finally, test-retest reliability over an interval of 4.5 months for the PP scale was 0.85 and internal consistency was $\mathbf{0 . 9 0}$. The high stability coefficient associated with the PP subscale suggests that it is a measure of a stable disposition as opposed to current mood state. For the FD subscale, the test-retest coefficient was $\mathbf{0 . 7 8}$ and internal consistency was $\mathbf{0 . 8 6}$. Correlation of the depression subscale with another personality inventory, the Minnesota Multiphasic Personality Inventory (MMPI) and the PP subscale of the MBHI was $0 \cdot 56$. Other validity coefficients for the PP subscale range from 0.50 to 0.66 . $^{12}$ For the FD subscale, correlation with the MMPI depression scale is $\mathbf{0 . 5 1}$. Other validity coefficients for the FD subscale range from 0.47 to 0.58 .

Subjects were seated in a room adjacent to the examiner and given as much time as needed to complete the two depression indices.

\section{Results}

The Parkinson's disease and control groups were compared on level of depression using the BDI and the FD and PP subscales of the MBHI. Results indicated significant differences between groups on all three measures, with the Parkinson's disease patients
Table 1 Beck Item Means for Parkinson's disease and control groups

\begin{tabular}{|c|c|c|}
\hline Item & Parkinson's disease group & Control group \\
\hline $\begin{array}{r}1 \\
2 \\
3 \\
4 \\
5 \\
6 \\
7 \\
8 \\
9 \\
10 \\
11 \\
12 \\
13 \\
14 \\
15^{*} \\
16^{*} \\
17^{*} \\
18^{*} \\
19^{*} \\
20^{*} \\
21^{*}\end{array}$ & $\begin{array}{l}0.37 \\
0.56 \\
0.36 \\
0.47 \\
0.27 \\
0.32 \\
0.26 \\
0.41 \\
0.29 \\
0.47 \\
0.87 \\
0.37 \\
0.62 \\
0.64 \\
1.05 \\
1.10 \\
1.10 \\
0.38 \\
0.55 \\
0.87 \\
0.97\end{array}$ & $\begin{array}{l}0.10 \\
0.19 \\
0.15 \\
0.24 \\
0.07 \\
0.12 \\
0.16 \\
0.18 \\
0.03 \\
0.22 \\
0.57 \\
0.18 \\
0.21 \\
0.29 \\
0.57 \\
0.74 \\
0.68 \\
0.10 \\
0.13 \\
0.25 \\
0.96\end{array}$ \\
\hline $\begin{array}{l}\text { BDI } \\
\text { MBHI (PP) } \\
\text { MBHI (FD) }\end{array}$ & $\begin{array}{l}11 \cdot 6 \\
55 \cdot 9 \\
62 \cdot 7\end{array}$ & $\begin{array}{r}6 \cdot 1 \\
41 \cdot 8 \\
52 \cdot 1\end{array}$ \\
\hline
\end{tabular}

*Represents a somatic item.

reporting higher levels of depression $(t(160)=5.08, p$ $<0.001$ for BDI; $t(120)=2.96, p<0.005$ for FD; $t(120)=3.55, p<0.001$ for PP) than controls (table 1).

Mean item scores on the BDI were calculated for the Parkinson's disease and control groups separately (table 1). Somatic items are identified in the table with an "*". For both Parkinson's disease and control groups, the items having the four highest mean scores were in the somatic category.

Internal consistency reliability estimates were calculated on the BDI as well as the NS and S subscales. The resulting coefficients are presented in table 2 for each group separately. The results indicate acceptable levels of reliability $(>0.80)$ for both the BDI and the NS subscale. Therefore, when somatic items were dropped from the BDI, internal consistency reliability did not improve in a meaningful way. This was particularly true for the Parkinson's disease group. The S subscale did not represent an internally consistent measure in either group. Higher estimates were

Table 2 Internal consistency reliability estimates for Parkinson's disease and control groups

\begin{tabular}{lll}
\hline Scale & Parkinson's disease group & Control group \\
\hline BDI & 0.88 & 0.75 \\
NS & 0.89 & 0.79 \\
S & 0.62 & 0.40 \\
\hline
\end{tabular}


Table 3 Factor loadings for Parkinson's disease and control groups

\begin{tabular}{lll}
\hline Scale & Parkinson's disease group & Control group \\
\hline NS & 0.53 & 0.61 \\
S & 0.53 & 0.43 \\
PP & 0.90 & 0.89 \\
FD & 0.85 & 0.82 \\
\hline
\end{tabular}

obtained in the Parkinson's disease group than in the control group for all measures studied.

A principal axis factor analysis of the S, NS, PP, and FD subscales was conducted on each group separately to determine whether the BDI derived subscales relate to other measures of depression in the Parkinson's disease sample in the same way as in the control group. One hundred and six subjects in the Parkinson's disease sample and 62 subjects in the control group had complete data on all four measures. In each group, there was only one eigenvalue greater than unity (2.48 and 2.42 for Parkinson's disease and control groups, respectively), and its associated factor explained approximately $61 \%$ of the variance. The factor loadings for each group are reported in table 3. It is seen that the loadings are quite similar between groups for all measures.

The last analysis conducted consisted of a principal axis factor analysis on 97 Parkinson's disease patients with complete data on the MBHI (PP and FD), BDI (NS and S) and the motor indices (R, B, and T) from the neurological examination. The purpose of this analysis was to determine whether two factors, depression and Parkinson's disease symptoms, could explain the intercorrelations among these measures, with the depression subscales (NS, PP, and FD) loading on the first factor and the somatic items (S) and Parkinson's disease symptoms ( $R, B$, and $T)$ on the second factor.

Although three eigenvalues were greater than unity, a scree plot indicated that two factors provided a satisfactory solution and were rotated to varimax criterion. Factor loadings greater than $\mathbf{0 . 3 0}$ are shown in table 4. It is evident from the loadings that the depression subscales define the first factor. The rigidity and bradykinesia measures define Factor 2 . Relevant to our study is the finding that the somatic sub-

Table 4 Factor loadings for Parkinson's disease group

\begin{tabular}{lll}
\hline Scale & Factor 1 & Factor 2 \\
\hline PP & 0.89 & - \\
FD & 0.80 & - \\
NS & 0.54 & - \\
S & 0.58 & - \\
Tremor & - & 0.96 \\
Rigidity & - & 0.60 \\
Bradykinesia & - & \\
\hline
\end{tabular}

scale loaded on the depression factor and not on Factor 2.

\section{Discussion}

The assessment of depression in medical populations presents a unique problem to researchers who use instruments that are normed on psychiatric subjects. Patients with chronic progressive illnesses such as Parkinson's disease exhibit a wide range of symptoms which are not necessarily shared by psychiatrically impaired but physically healthy adults. As a result, when measuring an affective construct with somatic symptoms as part of its operational definition, as is the case when measuring depression in Parkinson's disease patients, physical manifestations of the disease are confounded with affective status.

The difficulty in discriminating between somatic depressive symptoms and true depression is not unique to Parkinsonian patients. This situation occurs in other medical populations with chronic progressive diseases such as multiple sclerosis, amyotrophic lateral sclerosis, and cancer. ${ }^{13-15}$ Furthermore, studies such as those of Zemore and Eames ${ }^{15}$ have shown that somatic symptoms are more prevalent among healthy elderly individuals as compared to young adults. It follows that measuring depression in elderly persons with a chronic disease is particularly problematic.

This study was designed to assess the validity and internal consistency of the BDI when used on a sample of patients with Parkinson's disease. The primary purpose was to determine whether the somatic component of the BDI measured symptoms of the disease or manifestations of depression.

Our data confirm previous studies $2571016-19$ showing that Parkinson's disease patients exhibit significantly more depressive symptomatology than controls. The findings are also consistent with a recent study by Gotham et al $^{10}$ who reported that Parkinson's disease subjects tend to endorse somatic items as one part of their depressive symptomatology. In our study, although there was a tendency for both Parkinson's disease and control subjects to rate somatic items more strongly than nonsomatic ones, Parkinson's disease patients were still significantly more depressed than normals on both the BDI as well as two subscales of the MBHI. It is of note that the MBHI was developed for use on a medical population and does not contain somatic items. This suggests that depression in Parkinson's disease patients is not a somatic artifact.

The overall internal consistency of the BDI calculated for the Parkinson's disease group compares favourably with the internal reliability reported by Beck $^{20}$ in early psychometric studies. Our data suggest that the internal consistency is actually higher for 
Parkinson's disease subjects, indicating stronger item similarity for Parkinson's disease as compared with control subjects. In fact, when somatic items are omitted from the BDI, internal consistency does not improve in a meaningful way.

The first factor analysis showed that all measures of depression define one factor which is strikingly similar between the Parkinson's disease and control groups. This indicates that the BDI has factorial validity when measuring depression in Parkinson's disease as well as control subjects. If the somatic items had detracted from the construct of depression in the Parkinson's disease group only, we would have expected discrepant findings between the group factors.

Finally, the factor analysis of the depression measures, taken together with the motor disability symptoms, clearly showed the existence of two distinct factors: depression and Parkinson's disease symptomatology. This suggests that in Parkinsonian patients depression can be measured separately from symptoms of the disease. Unexpectedly, the somatic subscale did not load with the Parkinson's disease symptoms (Factor 2) but with the depression factor (Factor 1). These data demonstrate that the BDI including the somatic items is a reliable and valid measure of depression for Parkinson's disease patients as well as control subjects.

It is well recognised that the construct of depression includes a somatic component in its definition. The third edition of the Diagnostic and Statistical Manual of Mental Disorders (DSM-III) ${ }^{21}$ lists several specific somatic symptoms as part of its definition for both major depressive episodes as well as dysthymic disorders. Even in situations where physical symptoms confound a medical illness with depression, it is still relevant and appropriate to include the somatic symptoms in the operational definition of this psychological construct.

This study was supported by a grant from the National Parkinson Foundation.

\section{References}

1 Celesia GG, Wanamaker WM. Psychiatric disturbances in Parkinson's disease. Dis Nerv Syst 1972;33:577-83.

2 Mindham RHS. Psychiatric symptoms in parkinsonism. J Neurol Neurosurg Psychiatry 1970;33:188-91.

3 Steer RA, Beck AT, Garrison B. Applications of the Beck Depression Inventory. In: Sartorius N, Ban TA, eds. Assessment of Depression. New York: Springer Verlag, 1986:123-42.

4 Coppen A, Metcalfe M. Levodopa and L-trytophan therapy in Parkinsonism. Lancet 1972;i:654-8.

5 Mayeux R, Stern Y, Rosen J, Leventhal J. Depression, intellectual impairment and Parkinson's disease. Neurology 1981;31:645-50.

6 Direnfeld LK, Albert ML, Volicer L, Langlais PJ, Marquis J, Kaplan E. Parkinson's disease. The possible relationship of laterality to dementia and neurochemical findings. Arch Neurol 1984;41:935-41.

7 Santamaria J, Tolosa E, Valles A. Parkinson's disease with depression: A possible subgroup of idiopathic parkinsonism. Neurology 1986;36:1130-3.

8 Taylor AE, Saint-Cyr JA, Lang AE, Kenny FT. Parkinson's disease and depression: a critical re-evaluation. Brain 1986;109:279-92.

9 Moro DA, Yahr MD. Movement disorders in the psychiatric patient. Hosp Community Psychiatry 1984;35:377-83.

10 Gotham AM, Brown RG, Marsden CD. Depression in Parkinson's disease: a quantitative and qualitative analysis. $J$ Neurol Neurosurg Psychiatry 1986;49:381-9.

11 Beck AT, Ward CH, Mendelson M, Mock J, Erbaugh J. An inventory for measuring depression. Arch Gen Psychiatry 1961;4:561-71.

12 Millon T, Green CJ, Meagher RB. Millon Behavioral Health Inventory, Manual, Third Edition. Minneapolis: National Computer Systems, Interpretive Scoring Systems, 1982.

13 Leiber L, Plumb MM, Gerstenzang ML, Holland J. The communication of affection between cancer patients and their spouses. Psychosom Med 1976;38:379-89.

14 Plum MM, Holland J. Comparative studies of psychological function in patients with advanced cancer-1. Self-reported depressive symptoms. Psychosom Med 1977;39:264-76.

15 Zemore R, Eames N. Psychic and somatic symptoms of depression among young adults. Institutionalized aged and noninstitutionalized aged. $J$ Gerontol 1979;34:716-22.

16 Warburton JW. Depressive symptoms in Parkinson patients referred for thalamotomy. J Neurol Neurosurg Psychiatry 1967;30:368-70.

17 Marsh GG, Markham CH. Does levodopa alter depression and psychopathology in Parkinsonism patients? J Neurol Neurosurg Psychiatry 1973;36:925-35.

18 Horn S. Some psychological factors in parkinsonism. J Neurol Neurosurg Psychiatry 1974;37:27-31.

19 Robins AH. Depression in patients with parkinsonism. $\mathrm{Br} J$ Psychiatry 1976;128:141-5.

20 Beck AT. Depression: Causes and Treatment. Philadelphia: University of Pennsylvania Press, 1972.

21 Diagnostic and Statistical Manual of Mental Disorders, Third Edition. Washington DC: American Psychiatric Association, 1980. 